\title{
Global Practical Tracking by Output Feedback for Nonlinear Systems with Unknown Growth Rate and Time Delay
}

\author{
Xuehua Yan ${ }^{1}$ and Xinmin Song ${ }^{2}$ \\ ${ }^{1}$ School of Electrical Engineering, University of Jinan, Jinan, Shandong 250022, China \\ ${ }^{2}$ School of Information Science and Engineering, Shandong Normal University, Jinan, Shandong 250014, China \\ Correspondence should be addressed to Xuehua Yan; huaxue20@163.com
}

Received 28 June 2014; Accepted 7 August 2014; Published 3 September 2014

Academic Editor: Carlo Bianca

Copyright (c) 2014 X. Yan and X. Song. This is an open access article distributed under the Creative Commons Attribution License, which permits unrestricted use, distribution, and reproduction in any medium, provided the original work is properly cited.

\begin{abstract}
This paper is the further investigation of work of Yan and Liu, 2011, and considers the global practical tracking problem by output feedback for a class of uncertain nonlinear systems with not only unmeasured states dependent growth but also time-varying time delay. Compared with the closely related works, the remarkableness of the paper is that the time-varying time delay and unmeasurable states are permitted in the system nonlinear growth. Motivated by the related tracking results and flexibly using the ideas and techniques of universal control and dead zone, an adaptive output-feedback tracking controller is explicitly designed with the help of a new Lyapunov-Krasovskii functional, to make the tracking error prescribed arbitrarily small after a finite time while keeping all the closed-loop signals bounded. A numerical example demonstrates the effectiveness of the results.
\end{abstract}

\section{Introduction}

As well known that the presence of time delay has a significant effect on system performance, it often causes deterioration of control system performance and may induce instability, oscillation, and poor performance in a large number of important physical, industrial, and engineering problems involving [1] networked control systems, information, or energy transportation. Therefore, the study of time delay systems has important practical significance and has received much attention in recent years [1-18]. Generally speaking, control design methods for time delay systems can be classified into two categories: delay-dependent $[2-4,11]$ and delay-independent [5-10].

From the survey on the problems of delayed systems in [19], there still have been many research issues coming up in the control problems of delayed systems. In this paper, we are concerned with the practical tracking for a more general class of uncertain nonlinear systems in the following form. (The following notations will be used throughout this paper. $\mathbf{R}$ denotes the set of all real numbers. $\mathbf{R}^{+}$denotes the set of all nonnegative real numbers. $\mathbf{R}^{n}$ denotes the real $n$-dimensional space. For a given vector or matrix $X, X^{T}$ denotes its transpose; for any $x \in \mathbf{R}^{n},\|x\|_{1}$ denotes the 1norm; that is, $\|x\|_{1}=\left|x_{1}\right|+\cdots+\left|x_{n}\right| ;\|x\|$ denotes the Euclidean (or 2-) norm of vector $x$, and for the matrix $P$, we use $\|P\|$ to denote its norm induced by the 2-norm of the corresponding vector; for any $x \in \mathbf{R}^{n}$, there always holds $\|x\|_{1} \leq \sqrt{n}\|x\|$.) Consider

$$
\begin{array}{r}
\dot{\eta}_{i}=\eta_{i+1}+\psi_{i}(t, \eta)+\varphi_{i}(t-d(t), \eta(t-d(t))), \\
i=1, \ldots, n-1, \\
\dot{\eta}_{n}=u+\psi_{n}(t, \eta)+\varphi_{n}(t-d(t), \eta(t-d(t))), \\
y=\eta_{1}-y_{r},
\end{array}
$$

where $\eta=\left[\eta_{1}, \ldots, \eta_{n}\right]^{T} \in \mathbf{R}^{n}$ is the system state vector with the initial value $\eta_{0}=\eta(0) ; u \in \mathbf{R}, y \in \mathbf{R}$, and $t \mapsto y_{r}(t), t \in$ $\mathbf{R}^{+}$, are the control input, system output, and reference signal, respectively; $d(t): R \rightarrow[0, d]$ is the time-varying time delay satisfying $\dot{d}(t) \leq \gamma<1$ for a known constant $\gamma$; and $\psi_{i}: \mathbf{R}^{+} \times \mathbf{R}^{n} \rightarrow \mathbf{R}, \varphi_{i}: \mathbf{R}^{+} \times \mathbf{R}^{n} \rightarrow \mathbf{R}, i=1, \ldots, n$, are unknown functions but continuous in the first argument and locally Lipschitz in the second one. In what follows, suppose only the system output is measurable. 
The objective of the paper is to design an adaptive controller such that the resulting closed-loop system is welldefined and globally bounded on $\mathbf{R}^{+}$, and furthermore, for any prescribed tracking precision $l>0$ and every initial condition, there is a finite time $T_{\lambda}>0$ such that $\sup _{t \geq T_{\lambda}}|y(t)|=\sup _{t \geq T_{\lambda}}\left|\eta_{1}(t)-y_{r}(t)\right| \leq l$ (as described in [20]). To make this possible, the following assumptions are imposed on system (1) and reference signal $y_{r}$.

Assumption 1. There exists an unknown constant $\theta_{1} \geq 0$ such that

$$
\left|\psi_{i}(t, \eta)\right| \leq \theta_{1}\left(\left|\eta_{1}\right|+\cdots+\left|\eta_{i}\right|\right)+\theta_{1}, \quad i=1, \ldots, n .
$$

Assumption 2. There exists an unknown constant $\theta_{2} \geq 0$ such that

$$
\begin{array}{r}
\left|\varphi_{i}(t-d(t), \eta(t-d(t)))\right| \\
\leq \theta_{2}\left(\left|\eta_{1}(t-d(t))\right|+\cdots+\left|\eta_{i}(t-d(t))\right|\right)+\theta_{2}, \\
i=1, \ldots, n .
\end{array}
$$

Assumption 3. The reference signal $y_{r}$ is continuously differentiable, and moreover, there is an unknown constant $M \geq 0$ such that

$$
\sup _{t \geq 0}\left(\left|y_{r}(t)\right|+\left|\dot{y}_{r}(t)\right|\right) \leq M
$$

From Assumptions 1-3, it can be seen that the system investigated is substantially different from those of closely related tracking work $[4,10,20]$ since the system considered in this paper contains not only the time delay term but also the unmeasured state dependent growth. In fact, $[4,10]$ consider the state feedback tracking problem, and in both of those papers, the assumption on reference signal is stronger than Assumption 3 in the paper. Although [20] studies global practical tracking problem by output feedback, it does not include the time delay.

\section{Global Practical Tracking Control via Output Feedback}

In the section, we design an adaptive output-feedback tracking controller for system (1) satisfying Assumptions 1-3 and prove that, with unknown time-varying time delay $d(t)$ and unknown growth rate $\theta$ in Assumption 1 and without knowing the bound of the reference signal $y_{r}$ and its derivation $\dot{y}_{r}$ in Assumption 3, the global practical tracking for the systems (1) can also be achieved.

First, with the help of the coordinates transformation $x_{1}=$ $y, x_{i}=\eta_{i}, i=2, \ldots, n$, system (1) becomes

$$
\begin{aligned}
\dot{x}_{1}= & x_{2}+\phi_{1}\left(t, x, y_{r}, \dot{y}_{r}\right) \\
& +\varphi_{1}\left(t-d(t), x(t-d(t)), y_{r}(t-d(t))\right),
\end{aligned}
$$

$$
\begin{aligned}
\dot{x}_{i}= & x_{i+1}+\phi_{i}\left(t, x, y_{r}\right) \\
& +\varphi_{i}\left(t-d(t), x(t-d(t)), y_{r}(t-d(t))\right), \\
& i=2, \ldots, n-1, \\
\dot{x}_{n}= & u+\phi_{n}\left(t, x, y_{r}\right) \\
& +\varphi_{n}\left(t-d(t), x(t-d(t)), y_{r}(t-d(t))\right),
\end{aligned}
$$

where $\phi_{1}(\cdot)=\psi_{1}\left(t, x_{1}+y_{r}, x_{2}, \ldots, x_{n}\right)-\dot{y}_{r}, \phi_{i}(\cdot)=\psi_{i}\left(t, x_{1}+\right.$ $\left.y_{r}, x_{2}, \ldots, x_{n}\right), i=2, \ldots, n$.

By Assumptions 1 and 2 , for $i=1, \ldots, n$, it is easy to get the following inequalities:

$$
\begin{gathered}
\left|\phi_{i}\right| \leq \theta_{1}\left(\left|x_{1}\right|+\left|x_{2}\right|+\cdots+\left|x_{i}\right|\right)+\theta_{3}, \\
\left|\varphi_{i}\left(t-d(t), x(t-d(t)), y_{r}(t-d(t))\right)\right| \\
\leq \theta_{2}\left(\left|x_{1}(t-d(t))\right|+\left|x_{2}(t-d(t))\right|\right. \\
\left.+\cdots+\left|x_{i}(t-d(t))\right|\right)+\theta_{4},
\end{gathered}
$$

where $\theta_{3}=\theta_{1}(M+1)+M>0$ and $\theta_{4}=\theta_{2}(M+1)>0$ are unknown constants.

Then, motivated by [20], for any pregiven $l>0$, we still construct the following adaptive tracking controller for system (5):

$$
\begin{gathered}
\dot{\hat{x}}_{i}=\widehat{x}_{i+1}+L^{i} a_{i}\left(y-\widehat{x}_{1}\right), \quad i=1, \ldots, n-1, \\
\dot{\hat{x}}_{n}=u+L^{n} a_{n}\left(y-\widehat{x}_{1}\right), \\
\dot{L}=\max \left\{\frac{2\left(y-\widehat{x}_{1}\right)^{2}+2 \widehat{x}_{1}^{2}-\left(\lambda^{2} / 2\right)}{L^{2}}, 0\right\} \quad \text { with } L(0)=1,
\end{gathered}
$$

$$
u=-\left(L^{n} k_{1} \widehat{x}_{1}+L^{n-1} k_{2} \widehat{x}_{2}+\cdots+L k_{n} \widehat{x}_{n}\right),
$$

where $a_{i}>0$ and $k_{i}>0, i=1, \ldots, n$, are design parameters to be determined.

Similar to [20], before proving the claims of the theorem below, we first provide three fundamental propositions. The proof of Proposition 4 can be referred to Proposition 1 of [20]. The last two propositions are rigorously proven in Appendices A and B. Besides, by Proposition 4, it is not difficult to verify that the right-hand side of the resulting closed-loop system is continuous and locally Lipschitz in $(t, x, L)$ in an open neighborhood of the initial condition, and hence the closed-loop system has a unique solution on a small interval $\left[0, t_{s}\right)$ (see Theorem 3.1, page 18 of $\left.[21]\right)$. Let $\left[0, t_{f}\right)$ be its maximal interval on which a unique solution exists, where $0<t_{f}<+\infty$ (see Theorem 2.1, page 17 of [21]). 
Proposition 4. The gain $L$ determined by (8) is monotone nondecreasing on its existence interval, and its dynamics are locally Lipschitz in $\left(y, \widehat{x}_{1}, L\right)$.

Proposition 5. Define $e_{i}=x_{i}-\widehat{x}_{i}, \varepsilon_{i}=e_{i} / L^{i}$, and $z_{i}=\widehat{x}_{i} / L^{i}$, $i=1, \ldots, n$, and denote $\varepsilon=\left[\varepsilon_{1}, \ldots, \varepsilon_{n}\right]^{T}, z=\left[z_{1}, \ldots, z_{n}\right]^{T}$. Then, by choosing the suitable Lyapunov-Krasovskii functional $V(t, \varepsilon, z, L)$, there holds on $\left[0, t_{f}\right)$

$$
\dot{V} \leq-(L-\Theta)\left(\|\varepsilon\|^{2}+\|z\|^{2}\right)+\frac{\Theta}{L^{2}},
$$

where $\Theta$ is a positive constant.

Proposition 6. For the resulting closed-loop system, if $L$ is bounded on $\left[0, t_{f}\right)$, then $z$ and $\varepsilon$ are bounded on $\left[0, t_{f}\right)$ as well.

Remark 7. Definitions of $\varepsilon, z$ in Proposition 5 inspired by [20] are given the same as those in [20]. In fact, such definitions make it possible to offset the time delay term induced by the nonlinear time delay term $\varphi_{i}(\cdot)$ by skilly choosing a Lyapunov-Krasovskii functional and thereby still can obtain the similar result (inequality (10)), which plays a key role in the proof of the theorem below. In addition, since LyapunovKrasovskii functional method can provide less conservative and delay-independent results than Razumikhin theorem approach, we use Lyapunov-Krasovskii functional method to design the controller of system (1) in this paper.

Now, we are in a position to state the following theorem, to summarize the main results of the paper. For the proof of the theorem, the reader is referred to Theorem 1 in [20].

Theorem 8. Under Assumptions 1-3, the global practical output-feedback tracking problem of system (1) can be solved by the dynamic output-feedback controller of forms (8) and (9).

\section{An Illustrative Example}

This section gives a numerical example to illustrate the effectiveness of Theorem 8 .

Example 1. Consider the following uncertain nonlinear system:

$$
\begin{aligned}
& \dot{\eta}_{1}=\eta_{2}+\theta_{2} \eta_{1}(t-d(t)), \\
& \dot{\eta}_{2}=u-\theta_{1} \operatorname{sign}\left(\eta_{2}\right)\left(1+\left|\eta_{2}\right|\right), \\
& y=\eta_{1}-y_{r},
\end{aligned}
$$

where $d(t)=(1 / 2)(1+\sin (t))$ and $\operatorname{sign}(\cdot)$ denotes the signal function; that is, $\operatorname{sign}(x)=1(=-1)$ when $x>0(<0)$ and $\operatorname{sign}(x)=0$ when $x=0$; suppose $\theta=0.5, y_{r}=\sin (t)$.

Then a direct application of our proposed methodology yields a suitable adaptive output-feedback controller. Choose design parameters $a_{1}=1, a_{2}=10, k_{1}=12$, and $k_{2}=1$. Let the tracking accuracy be $\lambda=0.1$, and let initial conditions be $\eta_{1}(0)=0, \eta_{2}(0)=1, \widehat{x}_{1}(0)=2$, and $\widehat{x}_{2}(0)=-3$; we obtain Figures 1, 2, 3, 4, and 5 by numerical simulation.

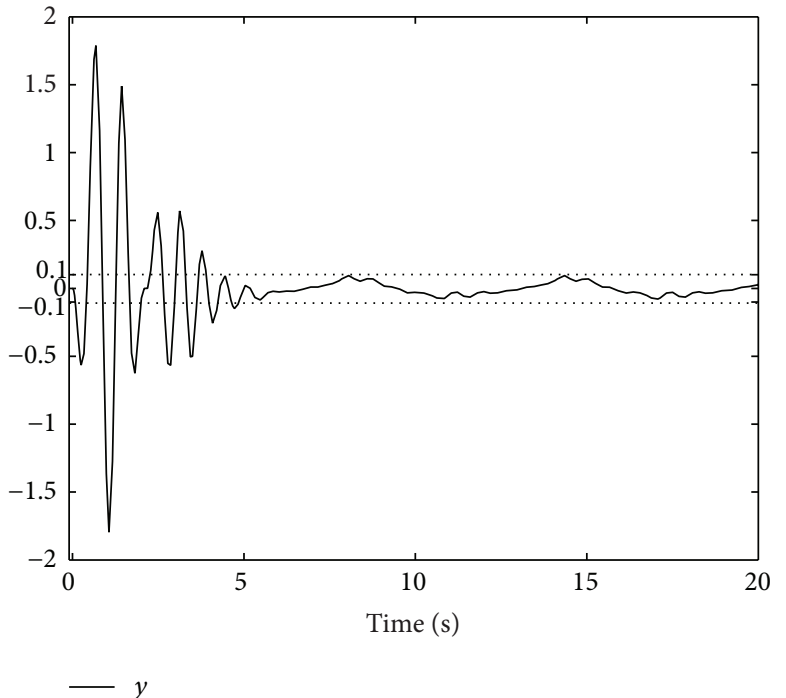

FIGURE 1: The trajectory of the tracking error $y$.

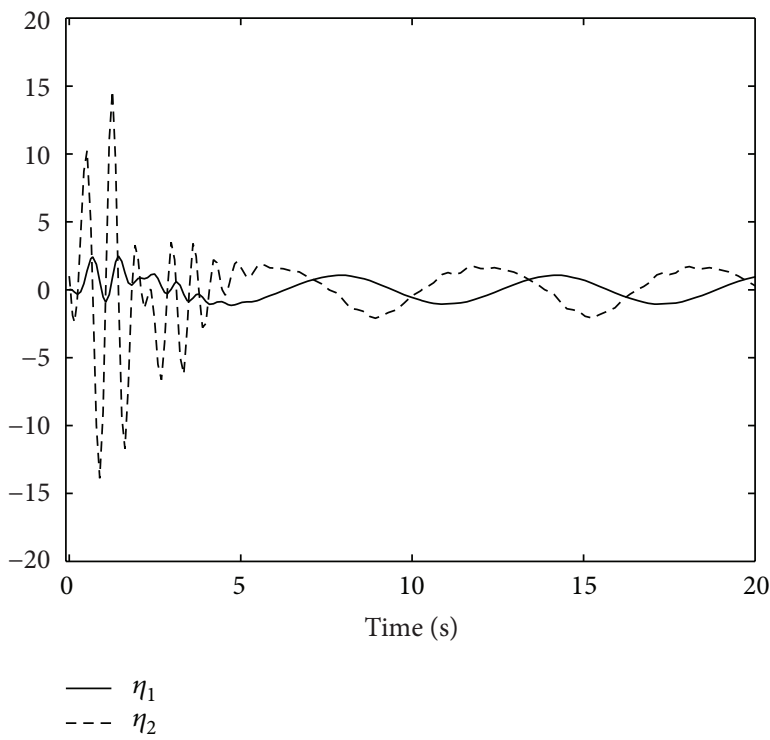

FIgURE 2: The trajectories of the system states $\eta_{1}$ and $\eta_{2}$.

From these figures, all the signals in the closed-loop system are bounded. From Figure 1, it can be seen that although the system contains time-varying time delay, after about seven seconds, the tracking error satisfies $|y|=\left|\eta_{1}-y_{r}\right| \leq 0.1$, which means that the prescribed tracking performance is achieved.

\section{Conclusions}

In this paper we extend the result in [20] to solve the global practical tracking problem for a class of nonlinear time delay systems by output feedback. Unlike most of the existing results, we allow the existence of unmeasurable states and time-varying time delay in the nonlinear growth. A stability analysis has been established based on the new LyapunovKrasovskii functional. The proposed controller independent 


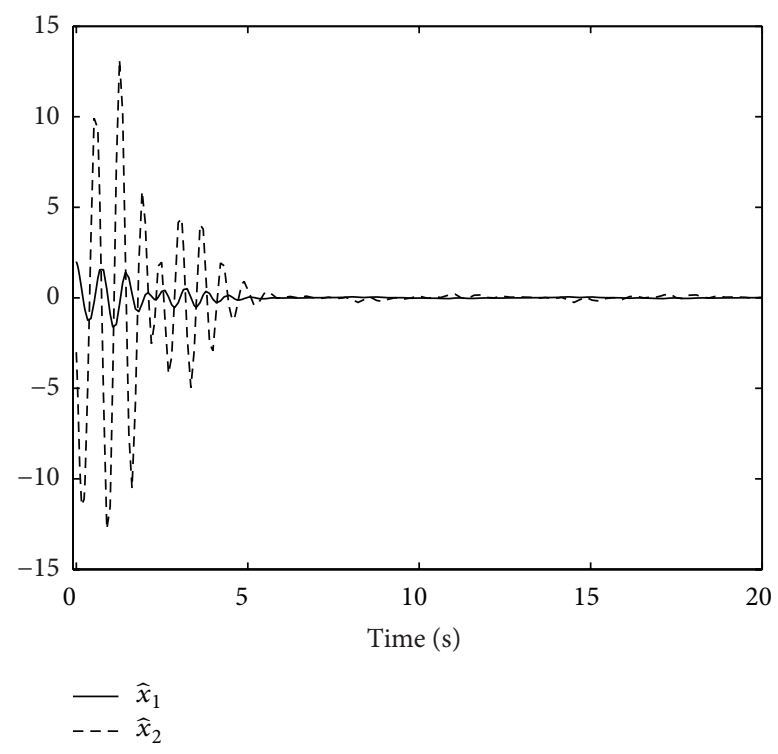

Figure 3: The trajectories of the observer states $\widehat{x}_{1}$ and $\widehat{x}_{2}$.

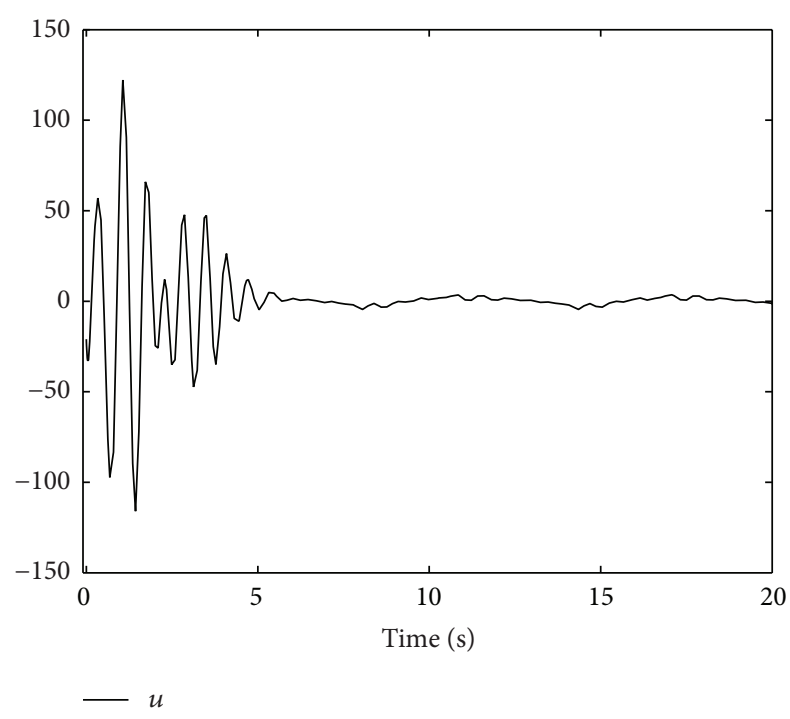

FIGURE 4: The trajectory of $u$.

of the derivative of time delay can make the tracking error arbitrarily small. Our future research is to extend the proposed framework for more general uncertain nonlinear systems, such as the systems with unknown control coefficients. Since the adopted controller in this paper is delayindependent, another topic of future work is to explore new delay-dependent, less conservative control design method.

\section{Appendices}

Appendices A and B provide the rigorous proofs of fundamental Propositions 5 and 6, which are collected here for the sake of compactness.

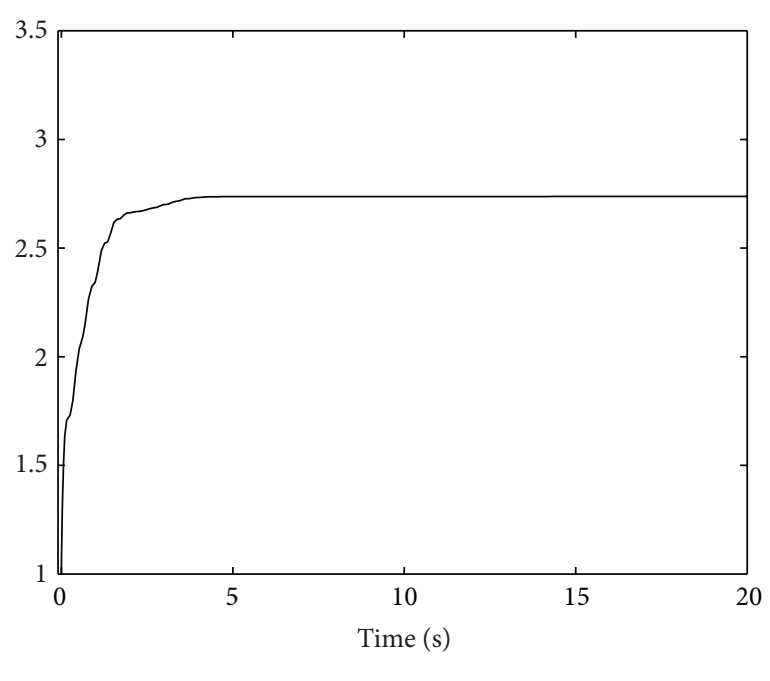

FIGURE 5: The trajectory of high-gain $L$.

\section{A. The Proof of Proposition 5}

With the aid of the definitions of $e_{i}$ 's, $\varepsilon_{i}$ 's, and $z_{i}$ 's and by (5) and (8), one has

$$
\begin{gathered}
\dot{e}_{i}=e_{i+1}-L^{i} a_{i} e_{1}+\phi_{i}(\cdot)+\varphi_{i}(\cdot), \quad i=1, \ldots, n-1, \\
\dot{e}_{n}=-L^{n} a_{n} e_{1}+\phi_{n}(\cdot)+\varphi_{n}(\cdot), \\
\dot{\varepsilon}=L A \varepsilon+\Phi\left(t, x, y_{r}, \dot{y}_{r}, L\right) \\
+\Psi\left(t-d(t), x(t-d(t)), y_{r}(t-d(t))\right)-\frac{\dot{L}}{L} D \varepsilon, \\
\dot{z}=L B z+L a \varepsilon_{1}-\frac{\dot{L}}{L} D z,
\end{gathered}
$$

where $x=\left[x_{1}, \ldots, x_{n}\right]^{T}, a=\left[a_{1}, \ldots, a_{n}\right]^{T}$, and $D=$ $\operatorname{diag}\{1,2, \ldots, n\}$,

$$
\begin{aligned}
\Phi & =\left[\frac{1}{L} \phi_{1}(\cdot), \frac{1}{L^{2}} \phi_{2}(\cdot), \ldots, \frac{1}{L^{n}} \phi_{n}(\cdot)\right]^{T}, \\
\Psi & =\left[\frac{1}{L} \varphi_{1}(\cdot), \frac{1}{L^{2}} \varphi_{2}(\cdot), \ldots, \frac{1}{L^{n}} \varphi_{n}(\cdot)\right]^{T}, \\
A & =\left[\begin{array}{cccc}
-a_{1} & 1 & \cdots & 0 \\
\vdots & \vdots & \ddots & \vdots \\
-a_{n-1} & 0 & \cdots & 1 \\
-a_{n} & 0 & \cdots & 0
\end{array}\right] \in \mathbf{R}^{n \times n},
\end{aligned}
$$

$$
B=\left[\begin{array}{cccc}
0 & 1 & \cdots & 0 \\
\vdots & \vdots & \ddots & \vdots \\
0 & 0 & \cdots & 1 \\
-k_{1} & -k_{2} & \cdots & -k_{n}
\end{array}\right] \in \mathbf{R}^{n \times n} \text {. }
$$


In terms of Lemma 1 in [22], suitable constants $a_{i}$ 's and $k_{i}$ 's can be chosen such that the matrices $A, B$ are Hurwitz ones and there exist $P=P^{T}>0$ and $Q=Q^{T}>0$ satisfying

$$
\begin{array}{cc}
A^{T} P+P A \leq-I, & D P+P D \geq 0, \\
B^{T} Q+Q B \leq-2 I, & D Q+Q D \geq 0 .
\end{array}
$$

Keeping this in mind, choose the Lyapunov-Krasovskii functional $V: \mathbf{R}^{n} \times \mathbf{R}^{n} \times \mathbf{R}^{+} \rightarrow \mathbf{R}^{+}$

$$
V(t, \varepsilon, z, L)=m V_{1}(\varepsilon)+V_{2}(z)+V_{3}(t, L)
$$

for system (A.2), where $m=\|Q a\|^{2}+1$ and $V_{i}, i=1,2,3$, defined by

$$
\begin{aligned}
V_{1}(\varepsilon) & =\varepsilon^{T} P \mathcal{E}, \\
V_{2}(z) & =z^{T} \mathrm{Q} z, \\
V_{3}(t, L) & =\frac{m\|P\|}{1-\gamma} \sum_{i=1}^{n} \int_{t-d(t)}^{t} \frac{1}{L^{2 i}(s)} \varphi_{i}^{2}\left(s, x(s), y_{r}(s)\right) \mathrm{d} s .
\end{aligned}
$$

Then, on the interval $\left[0, t_{f}\right)$, along the solutions of (A.2), the derivative of $V$ satisfies

$$
\begin{aligned}
\dot{V}= & -L m\|\varepsilon\|^{2}-m \frac{\dot{L}}{L} \varepsilon^{T}(D P+P D) \varepsilon-2 L\|z\|^{2} \\
- & \frac{\dot{L}}{L} z^{T}(D Q+Q D) z+2 m \varepsilon^{T} P \Phi+2 m \varepsilon^{T} P \Psi \\
+ & 2 L \varepsilon_{1} z^{T} Q a+\frac{m\|P\|}{1-\gamma} \\
\times & \sum_{i=1}^{n}\left(\frac{1}{L^{2 i}} \varphi_{i}^{2}-\frac{1}{L^{2 i}(t-d(t))}\right. \\
& \cdot \varphi_{i}^{2}\left(t-d(t), x(t-d(t)), y_{r}(t-d(t))\right) \\
& \times(1-\dot{d}(t))) .
\end{aligned}
$$

By supposition, $d(t) \in[0, d], \dot{d}(t) \leq \gamma<1$, and noticing that $L(t)$ is nondecreasing with $L \geq 1$, the following inequality holds:

$$
\begin{aligned}
& -\frac{m\|P\|}{1-\gamma} \frac{1}{L^{2 i}(t-d(t))} \varphi_{i}^{2} \\
& \quad \times\left(t-d(t), x(t-d(t)), y_{r}(t-d(t))\right)(1-\dot{d}(t)) \\
& \leq-m\|P\| \frac{1}{L^{2 i}(t)} \varphi_{i}^{2} \\
& \quad \times\left(t-d(t), x(t-d(t)), y_{r}(t-d(t))\right), \quad \forall t \in\left[0, t_{f}\right) .
\end{aligned}
$$

This, together with (A.4) and the fact that $\dot{L} \geq 0, L \geq 1$ on $\left[0, t_{f}\right)$, leads to

$$
\begin{aligned}
\dot{V} \leq & -L m\|\varepsilon\|^{2}-2 L\|z\|^{2}+2 m \varepsilon^{T} P \Phi \\
& +2 m \varepsilon^{T} P \Psi+2 L \varepsilon_{1} z^{T} Q a+\frac{m\|P\|}{1-\gamma} \sum_{i=1}^{n} \frac{1}{L^{2 i}} \varphi_{i}^{2} \\
& -m\|P\| \sum_{i=1}^{n} \frac{1}{L^{2 i}} \varphi_{i}^{2}\left(t, x(t-d(t)), y_{r}(t-d(t))\right) .
\end{aligned}
$$

By the method of completing square, we have

$$
\begin{gathered}
\left|2 m \varepsilon^{T} P \Phi\right| \leq 6 \theta m n\|P\|\left(\|\varepsilon\|^{2}+\|z\|^{2}\right)+\frac{4 \theta_{1}^{2} m\|P\|}{\theta L^{2}}, \\
\frac{m\|P\|}{1-\gamma} \sum_{i=1}^{n} \frac{1}{L^{2 i}} \varphi_{i}^{2} \leq \frac{4 \theta_{2}^{2} m n^{2}\|P\|}{1-\gamma}\left(\|\varepsilon\|^{2}+\|z\|^{2}\right)+\frac{2 \theta_{4}^{2} m n\|P\|}{(1-\gamma) L^{2}}, \\
\left|2 m \varepsilon^{T} P \Psi\right| \leq m\|P\|\left(\|\varepsilon\|^{2}+\|z\|^{2}\right) \\
+m\|P\| \sum_{i=1}^{n} \frac{1}{L^{2 i}} \varphi_{i}^{2} \\
\times(t-d(t), x(t-d(t)), \\
\left.y_{r}(t-d(t))\right), \\
2 L \varepsilon_{1} z^{T} Q a \leq L\|z\|^{2}+L\|Q a\|^{2}\|\varepsilon\|^{2} .
\end{gathered}
$$

Thus, setting $\Theta=\max \left\{(6 \theta n+1) m\|P\|+\left(4 \theta_{2}^{2} m n^{2}\|P\| /(1-\right.\right.$ $\left.\gamma)),\left(4 \theta_{1}^{2} m\|P\| / \theta\right)+\left(2 \theta_{4}^{2} m n\|P\| /(1-\gamma)\right)\right\}$, from these inequalities and (A.9), it follows that (10) holds on $\left[0, t_{f}\right)$, and this completes the proof of Proposition 5.

\section{B. The Proof of Proposition 6}

Notice that $L\left(t_{f}\right)=\sup _{0 \leq t<t_{f}} L(t)$ since $L$ is monotone nondecreasing, continuous, and bounded on $\left[0, t_{f}\right)$. Along the same line as A2 of [20], the boundedness of $z$ on $\left[0, t_{f}\right.$ ) is easily obtained. Then, let us show that $\varepsilon$ is bounded on $\left[0, t_{f}\right)$. To this end, we introduce the change of coordinates $\xi_{i}=e_{i} /\left(L^{*}\right)^{i}, i=1, \ldots, n$, where $L^{*}$ is a constant satisfying

$$
L^{*} \geq \max \left\{L\left(t_{f}\right),\left(6 \theta_{1} n+1\right)\|P\|+\frac{4 \theta_{2}^{2} n^{2}\|P\|}{1-\gamma}+3\right\} .
$$

Then, the error dynamics (A.1) is transformed into

$$
\begin{aligned}
\dot{\xi}= & L^{*} A \xi+L^{*} a \xi_{1}-L \Gamma a \xi_{1}+\Phi^{*}\left(t, x, y_{r}, \dot{y}_{r}\right) \\
& +\Psi^{*}\left(t-d(t), x(t-d(t)), y_{r}(t-d(t))\right),
\end{aligned}
$$

where $\Gamma=\operatorname{diag}\left\{1, L / L^{*}, \ldots,\left(L / L^{*}\right)^{n-1}\right\}, \Phi^{*}=\left[\phi_{1} / L^{*}\right.$, $\left.\phi_{2} /\left(L^{*}\right)^{2}, \ldots, \phi_{n} /\left(L^{*}\right)^{n}\right]^{T}$, and $\Psi^{*}=\left[\varphi_{1} / L^{*}, \varphi_{2} /\left(L^{*}\right)^{2}, \ldots\right.$, $\left.\varphi_{n} /\left(L^{*}\right)^{n}\right]^{T}$. 
For system (B.2), we choose

$$
\begin{aligned}
& V_{4}(t, \xi) \\
& \quad=\xi^{T} P \xi+\frac{\|P\|}{1-\gamma} \sum_{i=1}^{n} \int_{t-d(t)}^{t} \frac{1}{\left(L^{*}\right)^{2 i}} \varphi_{i}^{2}\left(s, x(s), y_{r}(s)\right) \mathrm{d} s .
\end{aligned}
$$

Similar to (A.8), one readily gets

$$
\begin{array}{r}
-\frac{\|P\|}{\gamma-1} \frac{1}{\left(L^{*}\right)^{2 i}} \varphi_{i}^{2}\left(t-d(t), x(t-d(t)), y_{r}(t-d(t))\right) \\
\times(1-\dot{d}(t)) \\
\leq-\|P\| \frac{1}{\left(L^{*}\right)^{2 i}} \varphi_{i}^{2}\left(t-d(t), x(t-d(t)), y_{r}(t-d(t))\right), \\
\forall t \in\left[0, t_{f}\right),
\end{array}
$$

and then, on $\left[0, t_{f}\right)$, differentiating function $V_{4}$ yields that

$$
\begin{aligned}
\dot{V}_{4} \leq & -L^{*}\|\xi\|^{2}+2 \xi_{1} L^{*} a^{T} P \xi-2 \xi_{1} L a^{T} \Gamma P \xi \\
+ & 2 \Phi^{* T} P \xi+2 \Psi^{* T} P \xi+\frac{\|P\|}{1-\gamma} \sum_{i=1}^{n} \frac{1}{\left(L^{*}\right)^{2 i}} \varphi_{i}^{2} \\
-\|P\| \sum_{i=1}^{n} & \frac{1}{\left(L^{*}\right)^{2 i}} \varphi_{i}^{2} \\
& \times\left(t-d(t), x(t-d(t)), y_{r}(t-d(t))\right) .
\end{aligned}
$$

With the definitions of $\varepsilon_{i}$ and $\xi_{i}$, using (6), (7), and (B.1), by the method of completing the square, we obtain

$$
\begin{gathered}
\left|2 \xi_{1} L^{*} a^{T} P \xi\right| \leq L^{* 2}\left\|a^{T} P\right\|^{2} \xi_{1}^{2}+\|\xi\|^{2} \\
\left|2 \xi_{1} L a^{T} \Gamma P \xi\right| \leq L^{2}\left\|a^{T} \Gamma P\right\|^{2} \xi_{1}^{2}+\|\xi\|^{2} \\
\left|2 \Psi^{* T} P \xi\right| \leq\|P\|\|\xi\|^{2}+\|P\| \\
\quad \times \sum_{i=1}^{n} \frac{1}{\left(L^{*}\right)^{2 i}} \varphi_{i}^{2}(t-d(t), x(t-d(t)), \\
\left.\quad y_{r}(t-d(t))\right) \\
\left|2 \Phi^{* T} P \xi\right| \leq 6 \theta_{1} n\|P\|\left(\|\xi\|^{2}+\|z\|^{2}\right)+\frac{4\|P\| \theta_{3}^{2}}{\theta_{1}}, \\
\frac{\|P\|}{1-\gamma} \sum_{i=1}^{n} \frac{1}{L^{2 i}} \varphi_{i}^{2} \leq \frac{4 \theta_{2}^{2} n^{2}\|P\|}{1-\gamma}\left(\|\xi\|^{2}+\|z\|^{2}\right)+\frac{2 \theta_{4}^{2} n\|P\|}{1-\gamma},
\end{gathered}
$$

which, together with (B.1), means that, on $\left[0, t_{f}\right)$,

$$
\dot{V}_{4} \leq-\frac{1}{\lambda_{\max }(P)} V_{4}+\theta_{5} \dot{L}+\theta_{5}\left(\sup _{0 \leq t<t_{f}}\|z(t)\|\right)^{2}+\theta_{5} \lambda^{2}+\theta_{5},
$$

where $\theta_{5}>0$ is a constant. From this, one easily gets that $\xi$ is bounded on $\left[0, t_{f}\right)$. Moreover, by the definitions of $\varepsilon_{i}$ and $\xi_{i}$ and the boundedness of $z$ (just proved), $\varepsilon$ is bounded on $\left[0, t_{f}\right)$.

\section{Conflict of Interests}

The authors declare that there is no conflict of interests regarding the publication of this paper.

\section{Acknowledgments}

This work is supported by the National Natural Science Foundation of China (nos. 61304013 and 61104126), the Doctoral Foundation of Jinan University (no. XBS1413), the Doctoral Fund of Ministry of Education of China (20113704120005), and the Excellent Young Scholars Research Fund of Shandong Normal University.

\section{References}

[1] S. L. Niculescu, Delay Effects on Stability: A Robust Control Approach, Springer, London, UK, 2001.

[2] E. T. Jeung, D. C. Oh, and H. B. Park, "Delay-dependent control for time-delayed T-S fuzzy systems using descriptor representation," International Journal of Control, Automation and Systems, vol. 2, no. 2, pp. 182-188, 2004.

[3] W. Chen, Z. Guan, and X. Lu, "Delay-dependent output feedback guaranteed cost control for uncertain time-delay systems," Automatica, vol. 40, no. 7, pp. 1263-1268, 2004.

[4] W. S. Chen and J. M. Li, "State feedback tracking control for nonlinear time-delay systems," Journal of Applied Sciences, vol. 24, no. 5, pp. 514-508, 2006.

[5] F. Mazenc, S. Mondie, and R. Francisco, "Global asymptotic stabilization of feedforward systems with delay in the input," IEEE Transactions on Automatic Control, vol. 49, no. 5, pp. 844850, 2004.

[6] S. K. Nguang, "Robust stabilization of a class of time-delay nonlinear systems," IEEE Transactions on Automatic Control, vol. 45, no. 4, pp. 756-762, 2000.

[7] Y. S. Fu, Z. H. Tian, and S. J. Shi, "Output feedback stabilization for time-delay nonlinear systems," Acta Automatica Sinica, vol. 28, no. 5, pp. 802-805, 2002.

[8] X. Zhang and Z. Cheng, "State feedback stabilization for a class of time-delay nonlinear systems," Acta Automatica Sinica. Zidonghua Xuebao, vol. 31, no. 2, pp. 287-290, 2005.

[9] H. Choi and J. Lim, "Output feedback regulation of a chain of integrators with an unknown time-varying delay in the input," IEEE Transactions on Automatic Control, vol. 55, no. 1, pp. 263268, 2010.

[10] Z. Mao and X. Xiao, "Decentralized adaptive tracking control of nonaffine nonlinear large-scale systems with time delays," Information Sciences, vol. 181, no. 23, pp. 5291-5303, 2011.

[11] W. Chen and J. Li, "Adaptive control for a class of nonlinear time-delay output-feedback systems," Control Theory and Applications, vol. 21, no. 5, pp. 844-847, 2004.

[12] T. Erneux, Applied Delay Differential Equations, Springer, New York, NY, USA, 2009.

[13] P. J. Cunningham and W. J. Wangersky, Time Lag in Population Models, Yale, 1958. 
[14] C. Bianca, M. Ferrara, and L. Guerrini, "The Cai model with time delay: existence of periodic solutions and asymptotic analysis," Applied Mathematics \& Information Sciences, vol. 7, no. 1, pp. 21-27, 2013.

[15] U. Fory and M. Bodnar, "Time delays in proliferation process for solid avascular tumour," Mathematical and Computer Modelling, vol. 37, no. 11, pp. 1201-1209, 2003.

[16] H. M. Byrne, "The effect of time delays on the dynamics of avascular tumor growth," Mathematical Biosciences, vol. 144, no. 2, pp. 83-117, 1997.

[17] C. Bianca and L. Guerrini, "On the Dalgaard-Strulik model with logistic population growth rate and delayed-carrying capacity," Acta Applicandae Mathematicae, vol. 128, pp. 39-48, 2013.

[18] M. J. Piotrowska, "Hopf bifurcation in a solid avascular tumour growth model with two discrete delays," Mathematical and Computer Modelling, vol. 47, no. 5-6, pp. 597-603, 2008.

[19] J. Richard, "Time-delay systems: an overview of some recent advances and open problems," Automatica, vol. 39, no. 10, pp. 1667-1694, 2003.

[20] X. Yan and Y. Liu, "Global practical tracking by output-feedback for nonlinear systems with unknown growth rate," Science China. Information Sciences, vol. 54, no. 10, pp. 2079-2090, 2011.

[21] J. K. Hale, Ordinary Differential Equations, Krieger, Huntington, NY, USA, 2nd edition, 1980.

[22] L. Praly and Z. P. Jiang, "Linear output feedback with dynamic high gain for nonlinear systems," Systems and Control Letters, vol. 53, no. 2, pp. 107-116, 2004. 


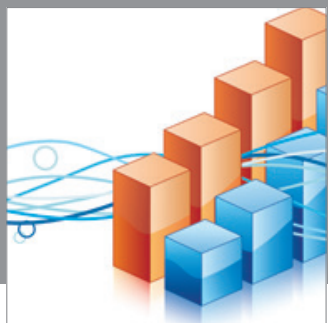

Advances in

Operations Research

mansans

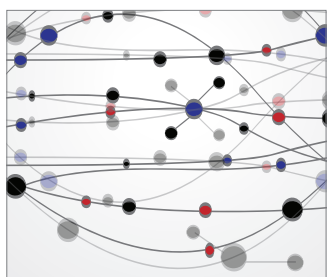

The Scientific World Journal
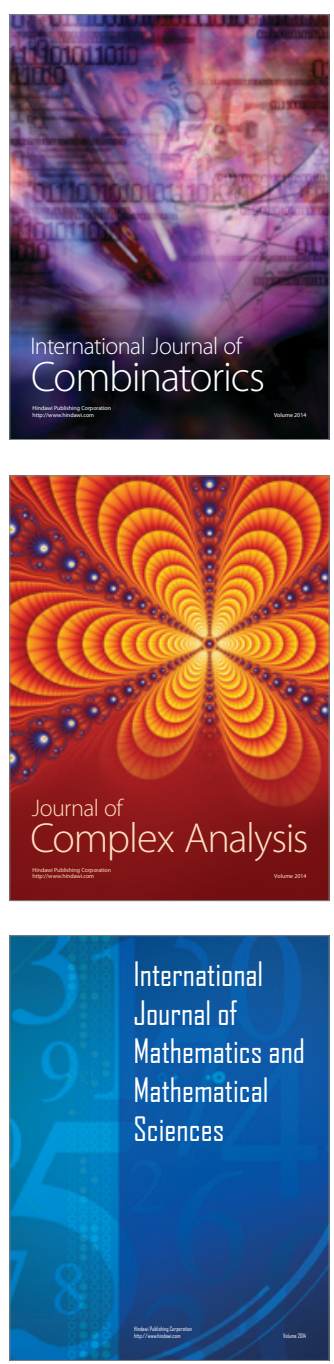
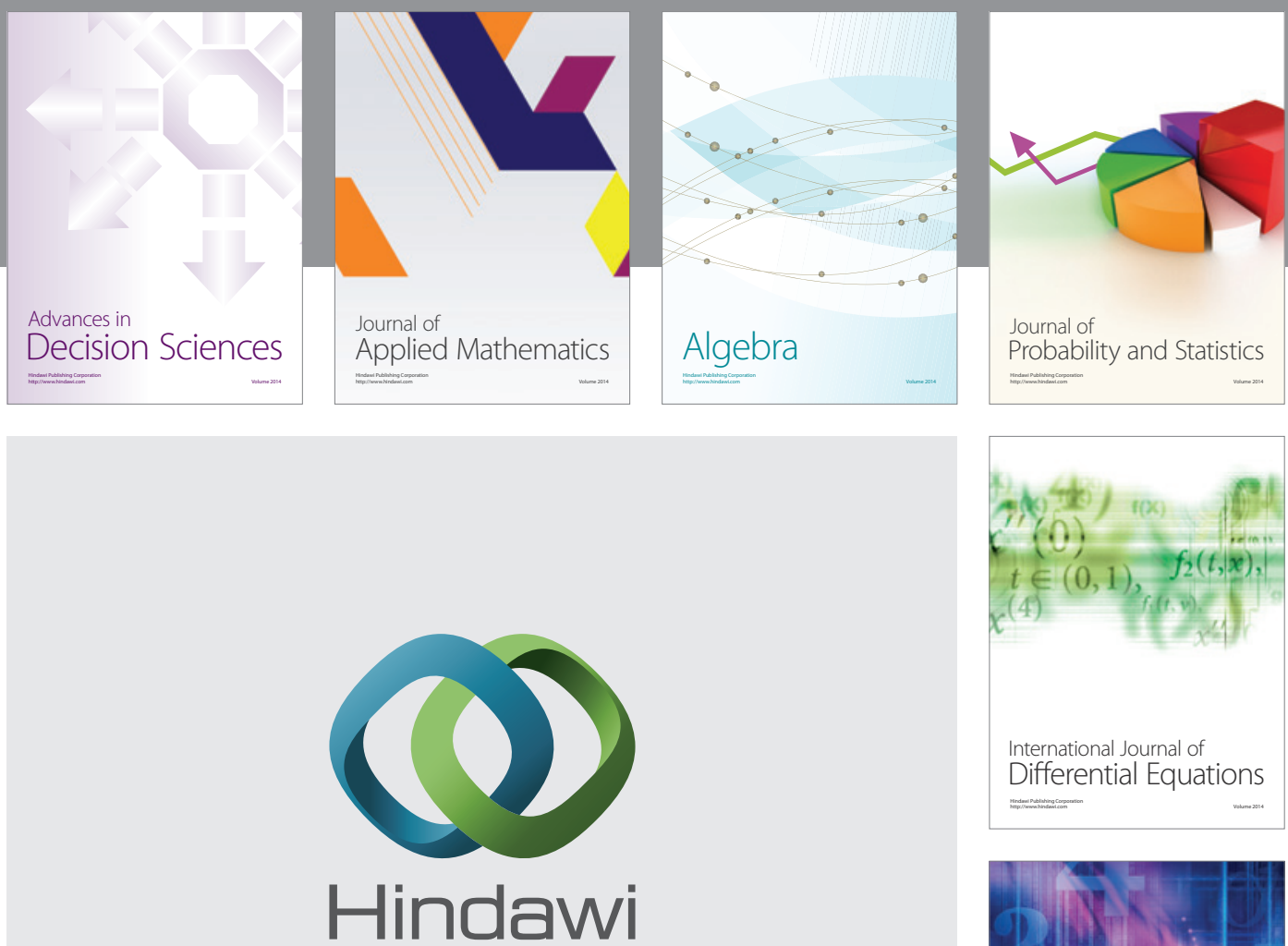

Submit your manuscripts at http://www.hindawi.com
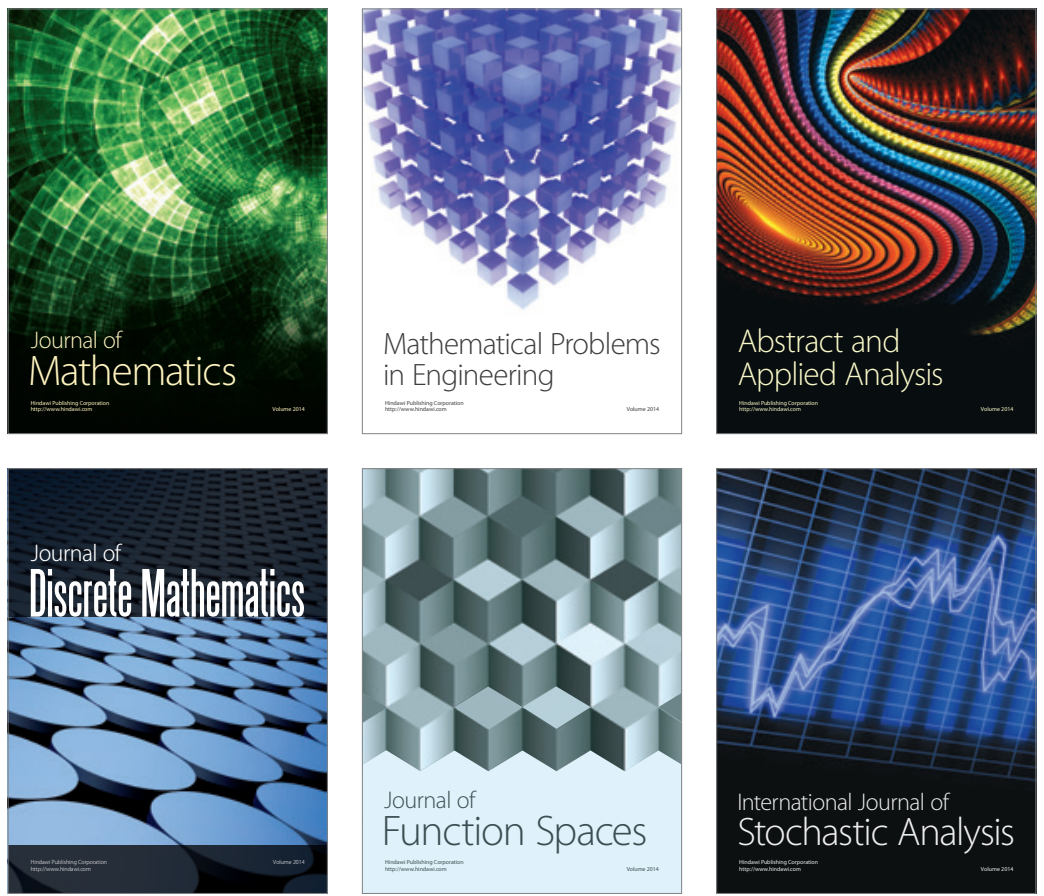

Journal of

Function Spaces

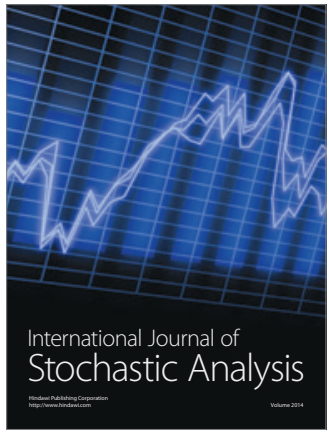

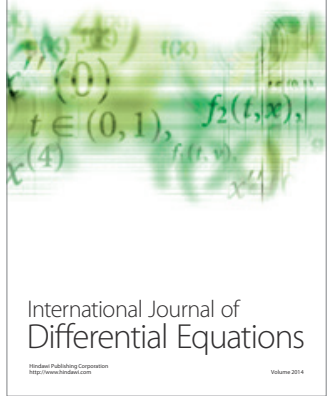
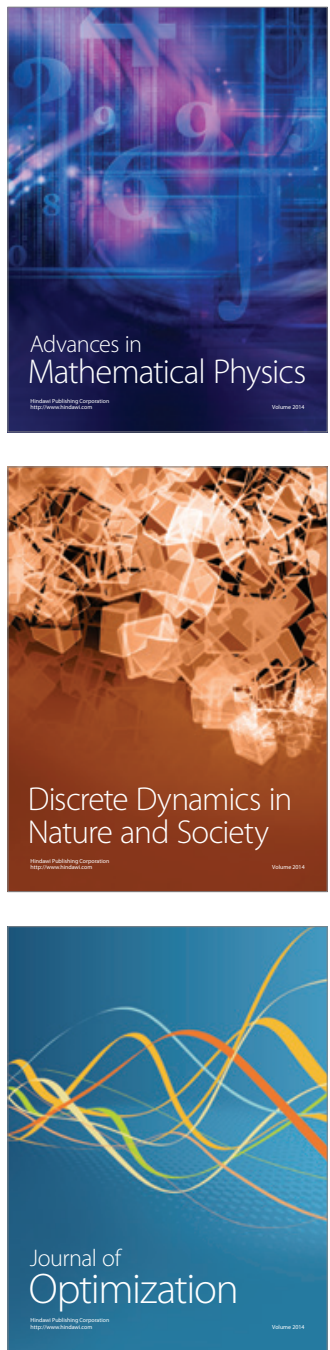